\title{
Modeling and control of water disinfection process in annular photoreactors
}

\author{
K. J. Keesman, D. Vries, S. van Mourik and H. Zwart
}

\begin{abstract}
As an alternative or addition to complex physical modeling, in this paper transfer function models of the disinfection process in annular photoreactors under different flow conditions are derived. These transfer function models allow an analytical evaluation of the system dynamics and the control strategies to gain further insight while preserving the physical process parameters. For diffusive flow conditions a dead-time/Padé approximation is proposed to find a low-order linear system description. Given the (approximate) transfer functions with their physical process parameters, an analytical feed-forward - feedback law is further worked out.
\end{abstract}

\section{INTRODUCTION}

$\mathrm{O}$ VER the years chlorination has been the most preferred disinfection process for water treatment. However, several investigations have proved that chlorine residuals are toxic to the aquatic life [1], while at the same time some by-products of chlorination have proved to be mutagenic. Therefore, the use of other disinfection techniques which are friendlier to the environment and do not arise health concerns is increasing.

It is known to scientists for nearly a century that ultraviolet (UV) light is an effective germicidal agent at certain wavelengths. However, the production cost of UV light was high. With the development of high intensity, long life lamps, interest in the use of UV as disinfection agent was renewed.

Precise modeling of the disinfection process in a UV photoreactor requires complex analysis of the radiation field [2]. This analysis needs to be linked to the modeling of the flow dynamics and the reaction kinetics. The models obtained are composed of very complicated differential equations which require demanding numerical computations (see e.g. [3]). Consequently, modeling of the disinfection process in a photoreactor is a quite complicated task. Moreover, phenomena such as reactivation of disinfected microorganisms make the situation even less

Manuscript received October 13, 2006. This work was supported by the Dutch organization for Scientific Research NWO, the Dutch Ministry of Economic Affairs and the technology foundation STW under the project number WWI.6345.

K. J. Keesman and D. Vries are with the Systems and Control Group, Wageningen University, Bornsesteeg 59, 6708 PD Wageningen, The Netherlands (corresponding author: K. J. Keesman, phone +31 317483780 ; fax +31 317 484957; e-mail: karel.keesman(wwr.nl).

S. van Mourik and H. Zwart are with the Department of Applied Mathematics, University of Twente, P.O. Box 217, 7500 AE Enschede, The Netherlands. straightforward. On the other hand, in practice simple models that preferably preserve prior knowledge are needed for fast online calculations.

The methods that have been used so far for the design of water disinfection systems are based on either complex physical models or empirical models. In this study our approach is to build relatively simple mathematical models based on the prior knowledge of the system. After setting up basic equations for the irradiation field, the effect of the type of flow is examined. Models are obtained for ideal plug flow as well as for diffusive flow. The ultimate goal of this paper is to show how to develop these relatively simple mathematical models that are suitable for dynamical analysis and control. Consequently, transfer functions are derived that connect the output of the system (bacteria load after disinfection) with the disturbance of system (initial load of bacteria) and the control inputs (light intensity and/or flow velocity).

In section 2 the UV disinfection process is described in some more detail. The modeling procedure of the disinfection process is presented in section 3. Section 4 presents two model approximation techniques. The resulting approximate models are used in section 5 to further derive an analytical feed-forward - feedback control law that explicitly depends on the physical process parameters.

\section{UV DISINFECTION}

A UV disinfection system transfers electromagnetic energy from a UV lamp to the genetic material of microorganisms. The absorption of light causes photochemical reactions that alter molecular components essential to cell function. There is scientific evidence to conclude that if sufficient dosages of UV energy reach the organisms, UV can disinfect water to whatever degree is required. In [4], the experimental data for UV inactivation of micro organisms have been extensively reviewed and furthermore they tabled the UV dose required to achieve the inactivation of bacteria, viruses and protozoa.

Predominantly, there are two types of UV sources that are used for water treatment, low pressure (LP) and medium pressure (MP) mercury lamps. The UV dose is the product of UV intensity $(\mathrm{mW} / \mathrm{cm} 2)$ and the average exposure time (s) of the water to be disinfected. In theory using a low intensity lamp for a longer period of time should give the same microbial inactivation as when a high intensity lamp is 
used for a shorter period. However, in [5] it is shown that preferably high intensity lamps should be used.

Absorption, reflection, refraction and scattering all interfere with the transportation of UV light. Reflection, refraction and scattering only change the direction of the light which is still capable of inactivating microorganisms, while absorbed light is no longer available.

The effectiveness of a system is related to the initial load of microorganisms in the water. In general, most of the disinfection models are based on the following expression:

$$
c=c_{0} \exp (-K t)
$$

where $c$ is the microbial load after disinfection (microorganisms $/ 100 \mathrm{~mL}$ ), $c_{0}$ the initial microbial load, $K$ the local inactivation rate constant $(1 / \mathrm{s})$ and $t$ the time of exposure (s). As can be seen from (1), inactivation of microorganism by UV irradiation is usually expressed in terms of first-order kinetics, which holds at low UV doses for e.g. vegetative bacteria as E. coli (see [5]).

\section{MODELING OF DISINFECTION PROCESS}

\section{A. Irradiation Field in Annular Reactor}

Analysis of light energy distribution in the annulus is important in order to determine the local inactivation rate constant. It becomes apparent that, since the amount of energy varies with space in the photoreactor, the same holds for the inactivation rate for the microorganisms.

For the development of a light distribution model for the annulus it is obvious that working in three dimensions leads to unnecessarily complex mathematical expressions. Therefore, it is assumed that both variations in light intensity in the longitudinal direction of the photoreactor and end effects of the lamp do not play a role. The developed model is also based upon the following assumptions:

- The UV lamp emits rays radially from the entire surface

- The attenuation of light depends on the concentration of solids in the medium and the length of the light path

- Solids are homogenously suspended in the medium, thus all the properties of the medium are assumed constant throughout the reactor

- There is monochromatic UV-light at $253.7 \mathrm{~nm}$ at which the DNA of all microorganisms is altered causing the inactivation of viruses and bacteria (see [6])

- The irradiation of the field is not time varying, it is only a function of the space coordinates of each point

- The effects of reflection and/or refraction are negligible

- There is only one species of microorganisms which follow first-order kinetics in the process of inactivation (see [7])

- Water has been pre-filtered, thus the concentration of suspended solids is small and irradiation field is only affected by the attenuation in water.

Under these assumptions and using Lambert's law, the light intensity at any point in the reactor is related to the surface flux (see [8]):

$$
\frac{1}{r} \frac{d(r I)}{d r}=-E I
$$

where $r$ is the radial distance in the reactor, $I$ is the light intensity of the irradiation field at a distance $r$ from the lamp $\left(\mathrm{mW} / \mathrm{cm}^{2}\right)$ and $E$ is the monochromatic absorbance of water $\left(\mathrm{cm}^{-1}\right)$. Integration of (2), using the boundary condition $I=I_{0}$ when $r=r_{0}$, gives:

$$
I=I_{0} \frac{r_{0}}{r} e^{-E\left(r-r_{0}\right)}
$$

where $I_{0}$ is the light intensity of the irradiation field on the surface of the UV-lamp $\left(\mathrm{mW} / \mathrm{cm}^{2}\right)$ and $r_{0}$ is the outer radius of the UV lamp $(\mathrm{cm})$. The reaction constant $(K)$ is the product of the available energy from the field multiplied by the susceptibility factor of the microorganism. Under the assumption that disinfection of a specific microorganism follows first-order kinetics we obtain:

$$
K(r)=\varepsilon I_{0} \frac{r_{0}}{r} e^{-E\left(r-r_{0}\right)}
$$

where $K(r)$ is the spatially dependent reaction constant $\left(\mathrm{s}^{-1}\right)$ and $\varepsilon$ is the susceptibility factor of the microorganism $\left(\mathrm{cm}^{2} /\right.$ $\mathrm{mW} \mathrm{s}$ ). The average light intensity related reaction constant across a cross-section of the tube will then be:

$$
K=I_{0} \frac{\varepsilon \int_{r_{0}}^{R} \frac{r_{0}}{r} e^{-E\left(r-r_{0}\right)} d r}{R-r_{0}}=I_{0} \beta
$$

Hence, the reaction constant $K$ depends on the light intensity on the surface of the lamp multiplied by the parameter $\beta$.

It is also possible to take into account the effects of reflection and refraction, see e.g. [6], [9] and [10], but then the reflection and refraction coefficient has to be identified in situ for each specific reactor.

\section{B. Flow in Annular Reactor}

In addition to the assumptions made in the previous section for the irradiation field, in order to develop the model for the case of ideal plug flow, the following assumptions have been made:

- The liquid is ideally mixed in the radial direction

- Every volume of the liquid has exactly the same retention time in the reactor 
- Every volume is receiving the same amount of radiation

- The only mechanism of mass transfer is convection (as yet, diffusion is neglected)

The equation that describes the disinfection process under the above assumptions is:

$$
\frac{\partial c(z, t)}{\partial t}+u_{z} \frac{\partial c(z, t)}{\partial z}+K c(z, t)=0
$$

After applying Laplace transformation with boundary condition at $z=0 \Rightarrow C(0, s)=C_{0}(s)$ the solution of the partial differential equation at $z=L$ is given by:

$$
C(L, s)=C_{0}(s) \mathrm{e}^{\frac{-(K+s)}{u_{z}} L}
$$

Consequently, the concentration at the end of the reactor is the output of the system, whereas the concentration at the entrance is the input. Therefore the above equation can be written in input-output form with transfer function $G(L, S)$ :

$$
G(L, s)=\frac{Y(s)}{U(s)}=\mathrm{e}^{\frac{-(K+s)}{u_{z}} L}
$$

Furthermore, we define: $t_{R}=L / u_{z}$ (residence time).

If, however, to be more realistic, we also assume diffusion in the $z$-direction with diffusion coefficient $\mathbf{D}$ the partial differential equation that describes this phenomenon is:

$$
\frac{\partial c(z, t)}{\partial t}+u_{z} \frac{\partial c(z, t)}{\partial z}-\mathbf{D} \frac{\partial^{2} c(z, t)}{\partial z^{2}}+K c(z, t)=0
$$

The solution of this differential equation, in terms of the Laplace variable $s$, is:

$$
C(z, s)=C_{1} e^{\frac{\frac{u_{z}}{\mathbf{D}}-\sqrt{\left(\frac{u_{z}}{\mathbf{D}}\right)^{2}+4\left(\frac{K+s}{\mathbf{D}}\right)}}{2}} z
$$

If we introduce the Peclet number, i.e. $P e=\frac{L u_{z}}{\mathbf{D}}$, and substitute the following boundary conditions: $C(z, 0)=0$, $C(0, s)=C_{0}(s)$ and $\lim _{z \rightarrow \infty} C(z, s)=0$, the transfer function $G(s)$ is given by:

$$
G(L, s)=e^{\frac{P e-\sqrt{P e^{2}+4 P e(\tilde{K}+s)}}{2}}
$$

with dimensionless reaction constant $\tilde{K}=K t_{R}$.

\section{MODEL APPROXIMATIONS}

\section{A. Padé approximation}

In the previous section transfer functions have been derived from partial differential equations. However, as it can be seen from (8) and (11) these transfer functions contain an exponential term in $s$. For further dynamical analysis or for controller design, preferably rational transfer functions (polynomial quotients in $s$ ) are required. Pure dead-time terms (of the form $\mathrm{e}^{-\tau s}$ ) are also allowed, because nowadays a vast amount of literature on so-called dead-time systems is available (see e.g. [11], [12] and the references therein). Consequently, (8) is a pure dead-time system with $\tau$ $=t_{R}$ and with a constant gain $\mathrm{e}^{-K t_{R}}$. However, for (11) with its square root of $s$ there is a need for a model approximation step. Instead of the commonly used Padé approximations, as in e.g. [13], we now derive a dead-time/Padé[0,1] approximation of (11), that is

$$
\tilde{G}(s)=e^{-\tau s} \frac{b}{s+a}
$$

(See Appendix A for details of this approximation). For $\mathrm{Pe}$ $=1000$ and $\tilde{K}=1$ Bode plots of the original and the approximate system with $a=22.43, b=8.36$ and $\tau=0.95$ are obtained (see Fig. 1), where the approximation is appropriate for a frequency smaller than $20 \mathrm{rad} / \mathrm{s}$.
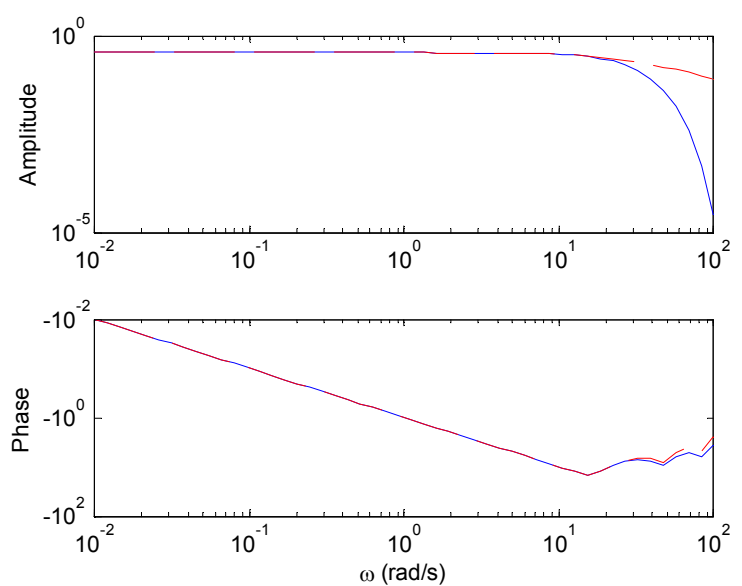

Figure 1. Comparison between original convectiondiffusion-reaction transfer function (blue line) and its deadtime/Padé[0,1] approximation (dashed red line).

\section{B. Linearization}

Notice that so far the transfer function between the disturbance input $C_{0}(s)$ and the concentration at the end of the reactor $C(L, s)$ for constant flow velocity and light intensity has been considered. For control of the disinfection process, in addition to possible shaping of the disturbance input by buffering, light intensity $\left(I_{0}\right)$, or in what follows $K$ $\left(=\beta I_{0}\right.$ with $\beta$ constant $)$, and flow velocity $\left(u_{z}\right)$ can be 
considered as control inputs or manipulated variables. For simplicity of the expressions only, in what follows we will focus on the ideal plug flow case; extension to diffusive flow in the $z$-direction is more or less straightforward. From (6) it follows that both control inputs appear in a bilinear form together with the concentration $c(z, t)$.

For small perturbations from the steady state (denoted by an overbar, e.g. $\bar{c}$ ) a linearized system description of the disinfection process can be obtained (see Appendix B for details). After some algebraic manipulations the following input-output relationship, relating the perturbed disturbance input $\Delta C_{0}$, the perturbed reaction constant $\Delta K=\beta \Delta I_{0}$ and the perturbed flow velocity $\Delta u_{z}$ to the perturbed system output $\Delta C(L, S)$, can be found:

$\Delta C(L, s)=G_{1}(L, s) \Delta C_{0}(s)+G_{2}(L, s) \Delta K(s)+G_{3}(L, s) \Delta u_{z}(s)$

Herein:

$$
\begin{aligned}
& G_{1}(L, s)=\mathrm{e}^{-\frac{L C_{1}}{\bar{u}_{z}}} e^{-\tau s} \text { with } C_{1}=\bar{K}=\overline{I_{0}} \beta \text { and } \tau=\frac{L}{\bar{u}_{z}} \\
& G_{2}(L, s)=-\frac{C_{2}}{s}+\frac{C_{2}}{s} \mathrm{e}^{-\tau s} \text { with } C_{2}=\bar{c}_{0} \mathrm{e}^{\frac{-L \bar{K}}{\bar{u}_{z}}} \\
& G_{3}(L, s)=-\frac{C_{3}}{s}+\frac{C_{3}}{s} \mathrm{e}^{-\tau s} \text { with } C_{3}=-\frac{\bar{c}_{0} \bar{K}}{\bar{u}_{z}} \mathrm{e}^{\frac{-L \bar{K}}{\bar{u}_{z}}}
\end{aligned}
$$

Consequently, the MISO system has three inputs and thus three transfer functions. Notice that $G_{1}(L, s)$ is a pure deadtime system with gain $\mathrm{e}^{-L \bar{K} / \bar{u}_{z}}$ and both $G_{2}(L, s)$ and $G_{3}(L, s)$ are parallel integrators with some time shift. In case of diffusive flow terms like in (13) will appear.

For $\bar{c}_{0}=0.75 \mathrm{~kg} / \mathrm{m}^{3}, \bar{K}=0.24 \mathrm{~s}^{-1}, L=5 \mathrm{~m}$ and $\bar{u}_{z}=1 \mathrm{~m} / \mathrm{s}$ the following step responses for the disturbance input and the light intensity related control input under ideal plug flow are presented in Fig. 2. From Fig. 2a it is immediately clear that after a dead time of $5 \mathrm{~s}$, the unit change in bacteria concentration at the entrance of the reactor (dotted line) is reduced to $30 \%$ of its initial value. Fig. $2 \mathrm{~b}$ shows that an increase of the light intensity initially reduces the bacteria concentration linearly with time and after $5 \mathrm{~s}$ a constant reduction is obtained. For use in a feed-forward controller design procedure (described in the next section), a step response of an approximate system of the form of (12), with $\tau=0, a=\frac{n \bar{u}_{z}}{L}(n=2)$ and $b=-\bar{c}_{0} e^{-\bar{K} L / \bar{u}_{z}}$, is also shown in Fig. $2 b$ (thin line).
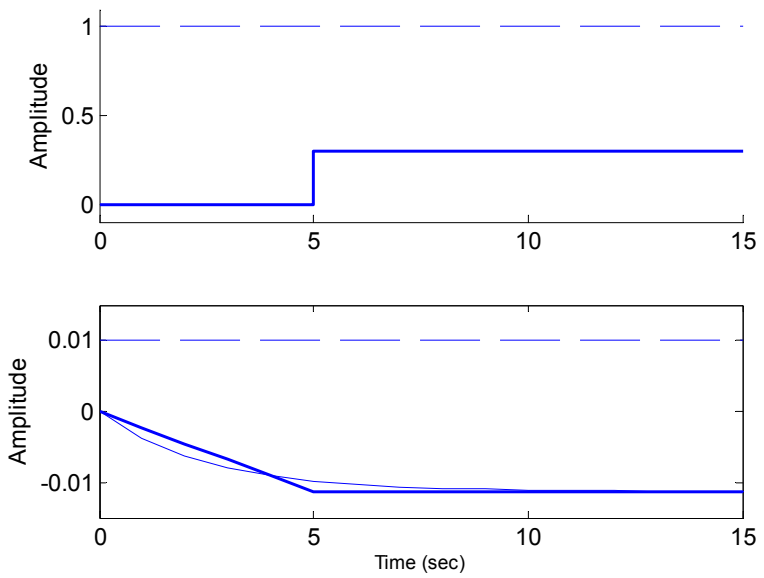

Figure 2. (a) Unit step $\left(\Delta c_{0}=1 \mathrm{~kg} / \mathrm{m}^{3}\right)$ response and (b) step $\left(\Delta K=0.01 \mathrm{~s}^{-1}\right)$ response (solid thin line: approximate system).

In the next section some suggestions for control of the disinfection process in the annular photoreactor are given and further analysed.

\section{PROCESS CONTROL DESIGN}

\section{A. Feed-forward control}

When the disturbance of a system is known or measured on-line, the use of a feed-forward controller can prove to be beneficial. The design of a feed-forward controller is rather simple but requires good models.

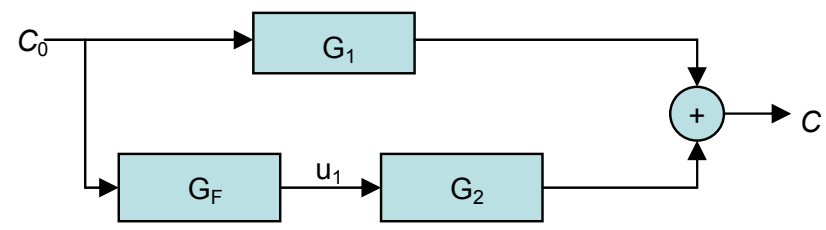

Figure 3. Feed-forward controller scheme for constant velocity $u_{z}$.

In Fig. 3 a feed-forward controller scheme for light intensity as control input is shown. Given the objective that the output should be close to zero, the design of the feedforward transfer function $G_{F}$ simply follows from the algebraic equation:

$$
C(L, s)=G_{1}(L, s) C_{0}+G_{F}(s) G_{2}(L, s) C_{0}=0
$$

so that $G_{F}(s)=-\frac{G_{1}(L, s)}{G_{2}(L, s)}$, where $G_{1}$ and $G_{2}$ follow from e.g. (14).

Let us evaluate the scheme in Fig. 3 for the ideal plug flow case with light intensity as control input in some more detail. 
In this case, in (15) $G_{1}$ is found from (14a) and $G_{2}$ from (14b). Consequently,

$$
G_{F}(s)=-\frac{G_{1}(L, s)}{G_{2}(L, s)}=\frac{s e^{-L s / \bar{u}_{z}}}{\bar{c}_{0}\left(1-e^{-L s / \bar{u}_{z}}\right)}
$$

which is a non-rational transfer function. Let us therefore use the approximation of $G_{2}$ presented in Fig. 2b. Then,

$$
\tilde{G}_{F}(s)=-\frac{G_{1}(L, s)}{\tilde{G}_{2}(L, s)}=\frac{e^{-L s / \bar{u}_{z}}}{\bar{c}_{0}} \frac{\left(s+n \bar{u}_{z} / L\right)}{\left(s+m \bar{u}_{z} / L\right)}
$$

where the factor $\left(s+m \bar{u}_{z} / L\right)$ with $m$ large is added to make this controller physically realizable. A similar filter term is usually added in the D-action of a PID controller. The overall transfer function $H(s)$ from $C_{0}$ to $C$ is then given by

$$
H(s)=e^{-\bar{K} L / \bar{u}_{z}}\left[1-\frac{\left(s+n \bar{u}_{z} / L\right)}{s\left(s+m \bar{u}_{z} / L\right)}\left(1-e^{-L s / \bar{u}_{z}}\right)\right] e^{-L s / \bar{u}_{z}}
$$

Fig. 4 presents the simulation results of the linearized system with the physically realizable feed-forward controller for $m=1000$ and as before for $\bar{c}_{0}=0.75 \mathrm{~kg} / \mathrm{m}^{3}, \bar{K}=0.24 \mathrm{~s}^{-1}$, $L=5 \mathrm{~m}$ and $\bar{u}_{z}=1 \mathrm{~m} / \mathrm{s}$. Since

$$
\begin{gathered}
\lim _{s \rightarrow 0} \frac{\left(1-e^{-L s / \bar{u}_{z}}\right)}{s}=\frac{L}{\bar{u}_{z}} \\
\Rightarrow \lim _{s \rightarrow 0} H(s)=e^{-\bar{K} L / \bar{u}_{z}}\left(1-\frac{n}{m} \cdot \frac{L}{\bar{u}_{z}}\right)
\end{gathered}
$$

Consequently, for the parameter values given above and as a result from the approximations, the deviation in the output at $z=L$ is equal to $0.03 \mathrm{~kg} / \mathrm{m}^{3}$ for $\Delta c_{0}=0.1 \mathrm{~kg} / \mathrm{m}^{3}$ and $t \rightarrow \infty$.
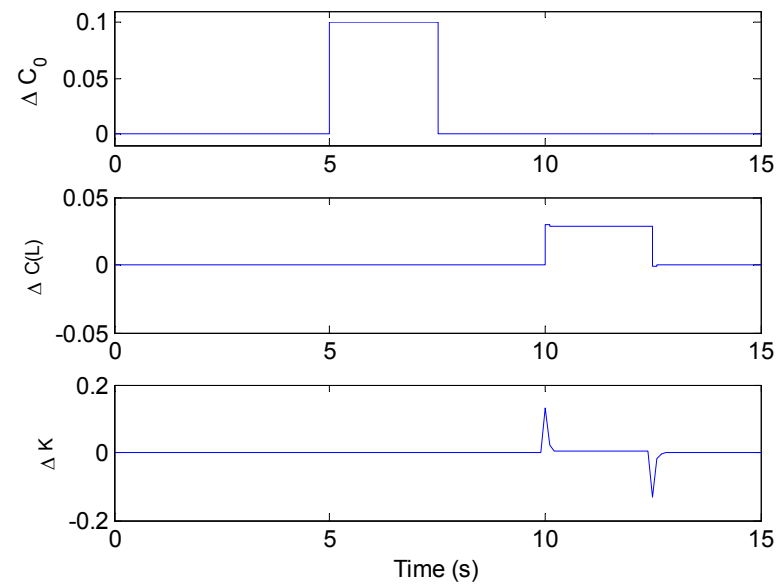

Figure 4. Simulation results for feed-forward control strategy.

\section{B. Combined feed-forward and feedback control}

Although feed-forward can theoretically result in perfect control of a process and perfect attenuation of known disturbances, in practice it is not always the case. That is mainly because it requires, as quoted before, very precise models. In practice, there are always deviations between the model and the real process and furthermore the measured or predicted disturbance input contains errors. Therefore the combination of feed-forward and feedback control can result in more precise control of the process (see e.g. [14]). For our application with constant flow velocity, Fig. 5 shows an appropriate feed-forward - feedback controller scheme.

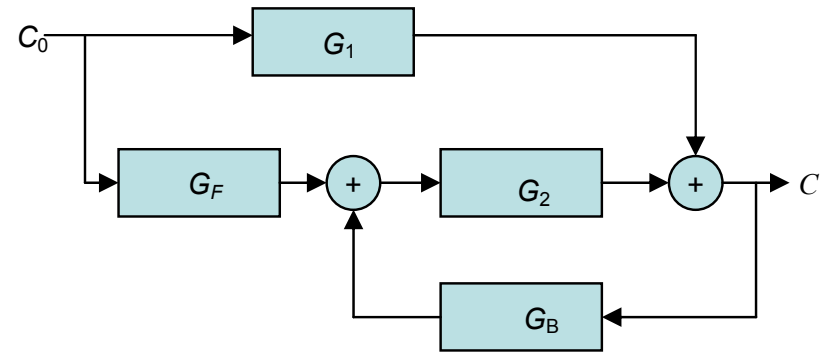

Figure 5. Feed-forward - feedback controller scheme.

The closed loop transfer function for the changes of the disturbance is:

$$
C(L, s)=\frac{G_{1}+G_{2} G_{F}}{1-G_{2} G_{B}} C_{0}(s)
$$

so that again $G_{F}(s)=-\frac{G_{1}(L, s)}{G_{2}(L, s)}$ or for implementation in practice $-\frac{G_{1}(L, s)}{\tilde{G}_{2}(L, s)}$. From $(21)$, however, it can be seen that the stability of the system and thus the denominator of the fraction, only depends on the process $G_{2}$ and the feedback controller $G_{B}$. This gives the opportunity to tune both controllers separately and deal with the stability of the system. The feedback controller $G_{B}$ can, for instance, take a PI-controller structure with controller parameters that directly depend on the dead-time first-order properties of $G_{2}$. Consequently,

$$
G_{B}(s)=K_{p}\left(1+\frac{1}{T_{r} s}\right)
$$

From the Cohen-Coon reaction curve method and after substituting from (12), for the ideal plug flow case, the time constant and gain, that is $a=\frac{n \bar{u}_{z}}{L}, b=-\bar{c}_{0} e^{-\bar{K} L / \bar{u}_{z}}$ and after choosing $\tau=\tau_{0}$, we obtain 


$$
K_{p}=-\frac{T e^{\bar{K} L / \bar{u}_{z}}}{\bar{c}_{0}}\left(0.9+\frac{1}{12 T}\right)
$$

and

$$
T_{r}=\tau_{0} \frac{(30 T+3)}{(9 T+20)}
$$

where $T=\frac{L}{n \bar{u}_{z} \tau_{0}}$. Hence, the controller parameters are fully expressed in terms of the process parameters. Again, from (21) the overall transfer function can be found. Fig. 6 presents the simulation results of the feed-forward feedback scheme (Fig. 5) for $n=2, m=1000, \bar{c}_{0}=0.75$ $\mathrm{kg} / \mathrm{m}^{3}, \bar{K}=0.24 \mathrm{~s}^{-1}, L=5 \mathrm{~m}$ and $\bar{u}_{z}=1 \mathrm{~m} / \mathrm{s}$.
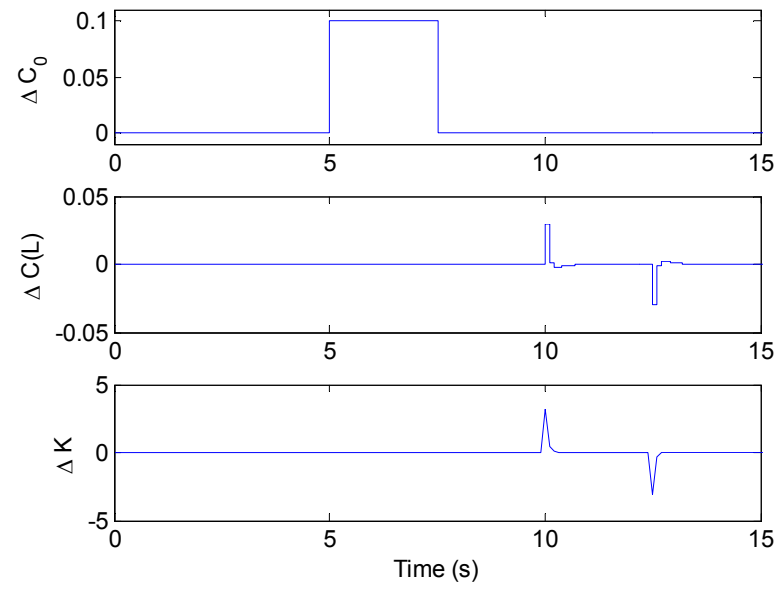

Figure 6. Simulation results for feed-forward - feedback control strategy.

Hence, these analyses show how prior non-rational process knowledge, which frequently appears in processes with flow components, can be directly implemented in a controller design procedure that conserve the knowledge of physical process parameters. Clearly, given the dynamical models of the previous sections, even a multivariable (optimal) controller could have been designed (see e.g. [11], [12]).

\section{CONCLUSIONS}

For dynamical analysis and model-based controller design of a water disinfection process in annular reactors, described by convection-diffusion-reaction type of differential equations, a transfer function modelling approach, using analytical expressions in terms of the Laplace variable $s$ and the original physical process parameters, is possible and provides further insight into the process (see also [13]).

\section{APPENDIX}

\section{A. Padé approximation}

A dead-time-Padé $[0,1]$ approximation of $G(s)$ in $s=0$ is of the form

$$
\tilde{G}(s)=e^{-\tau s} \frac{b}{s+a}
$$

where the coefficients $a, b$ and $\tau$ are determined by setting $G(0)=\tilde{G}(0), \quad \frac{d G(0)}{d s}=\frac{d \tilde{G}(0)}{d s} \quad$ and $\frac{d^{2} G(0)}{d s^{2}}=\frac{d^{2} \tilde{G}(0)}{d s^{2}}$.

Given the convection-diffusion transfer function $G(s)$ as in (11), we obtain (rather complicated) expressions for $a, b$ and $\tau$ in terms of $P e, \tilde{K}$ and $s$. However, the following relationships hold:

$$
\frac{b}{a}=e^{\frac{P e-\sqrt{P e^{2}+4 P e \tilde{K}}}{2}} \text { and } \tau+\frac{1}{a}=\frac{P e}{\sqrt{P e^{2}+4 P e \tilde{K}}}
$$

where $b / a$ is the steady-state gain of the system. This procedure can be repeated for different orders $m$ and $n$, but then most often we must rely on numerical schemes for the estimation of the coefficients. In general, an appropriate choice of the orders $n$ and $m$ in a Padé $[n, m]$ approximation is made by observation of the Bode plot of the original transfer function.

\section{B. Linearization}

Let us as an example of the linearization procedure write (6) in terms of the steady states (denoted by an overbar) and small perturbations, indicated by $\Delta$ :

$$
\begin{aligned}
& \frac{\partial(\bar{c}+\Delta c)}{\partial t}+\left(\bar{u}_{z}+\Delta u\right) \frac{\partial(\bar{c}+\Delta c)}{\partial z}+(\bar{K}+\Delta K)(\bar{c}+\Delta c)=0 \\
& \Rightarrow \frac{\partial \bar{c}}{\partial t}+\frac{\partial \Delta c}{\partial t}+\bar{u}_{z} \frac{\partial \bar{c}}{\partial z}+\bar{u}_{z} \frac{\partial \Delta c}{\partial z}+\Delta u_{z} \frac{\partial \bar{c}}{\partial z}+\Delta u_{z} \frac{\partial \Delta c}{\partial z} \\
& \quad+\bar{K} \bar{c}+\bar{K} \Delta c+\Delta K \bar{c}+\Delta K \Delta c=0
\end{aligned}
$$

Subtracting from (B.2) the steady state terms that obey (6) and neglecting the second-order terms $\Delta u_{z} \frac{\partial \Delta c}{\partial z}$ and $\Delta K \Delta c$ the following equation in the so-called deviation variables is obtained:

$$
\frac{\partial \Delta c}{\partial t}+\bar{u}_{z} \frac{\partial \Delta c}{\partial z}+\Delta u_{z} \frac{\partial \bar{c}}{\partial z}+\bar{K} \Delta c+\Delta K \bar{c}=0
$$

For both $\bar{u}_{z}$ and $\bar{K}$ the constant steady state values can be substituted, but for the concentration $\bar{c}$ the steady state solution must be found from: 


$$
\bar{u}_{z} \frac{d \bar{c}}{d z}+\bar{K} \bar{c}=0
$$

which is given by $\bar{c}=\bar{c}_{0} e^{-\bar{K} z / \bar{u}_{z}}$, so that in (B.3) $\frac{\partial \bar{c}}{\partial z}=-\frac{\bar{K} \bar{c}_{0}}{\bar{u}_{z}} e^{-\bar{K} z / \bar{u}_{z}}$. Hence, after substitution of the steady state solutions and after defining $u_{1}:=\Delta K$ and $u_{2}:=\Delta u_{z}$, (B.3) becomes

$$
\frac{\partial \Delta c}{\partial t}+\bar{u}_{z} \frac{\partial \Delta c}{\partial z}-\frac{\bar{K} \bar{c}_{0}}{\bar{u}_{z}} e^{-\bar{K} z / \bar{u}_{z}} u_{2}+\bar{K} \Delta c+\bar{c}_{0} e^{-\bar{K} z / \bar{u}_{z}} u_{1}=0 \text { (B.5) }
$$

After Laplace transformation and re-ordering the equation we obtain:

$$
\frac{\partial \Delta C}{\partial z}+\frac{(s+\bar{K})}{\bar{u}_{z}} \Delta C-\frac{\bar{K} \bar{c}_{0}}{\bar{u}_{z}^{2}} e^{-\bar{K} z / \bar{u}_{z}} U_{2}+\frac{\bar{c}_{0}}{\bar{u}_{z}} e^{-\bar{K} z / \bar{u}_{z}} U_{1}=0
$$

which is a linear first-order equation with the initial condition: $\Delta C(0)=\Delta C_{0}$, the disturbance input of the reactor system and $U_{1}=\Delta K(s), U_{2}=\Delta u_{z}(s)$ the control inputs. After solving (B.6) for $z=L$, the transfer functions $G_{1}(L, S)$ to $G_{3}(L, s)$ in (14) are obtained.

\section{REFERENCES}

[1] R. W. Ward and G. M. DeGrave, Residual toxicity of several disinfectants in domestic wastewater. Journal of Water Pollution Control Federation, 50(1): 46-60, 1978.

[2] A. E. Cassano, C. A. Martin, R. J. Brandi and O. M. Alfano, Photoreactor analysis and design: Fundamentals and applications. Industrial and Engineering Chemistry Research, 34: 2155-2201, 1995.

[3] L. G. Puma and P. L. Yue, Modeling and design of thin-film slurry photocatalytic reactors for water purification. Chemical Engineering Science, 58(11): 2269-2281, 2003.

[4] H. B. Wright and G. Sakamoto, UV dose required to achieve incremental log inactivation of bacteria, viruses, and protozoa. Trojan Tech Inc, revision of Sept 12, 2001.

[5] I. Woitenko, M. K. Stinson and R. Field, Challenges of Combined Sewer Overflow Disinfection by Ultraviolet Light Irradiation. Critical Reviews in Environmental Science and Technology, 31(3): 223-239, 2000.

[6] J. R. Bolton, Calculation of ultraviolet fluence rate distributions in an annular reactor: Significance of refraction and reflection. Water Research, 34: 3315-3324, 2000.

[7] B. F. Severin, M. T. Suidan, B. E. Rittmann and R. S. Engelbrecht, Inactivation kinetics in a flowthrough UV reactor. Joumal of the Water Pollution Control Federation 56: 164-169, 1984.

[8] M. T. Suidan and B. F. Severin, Light intensity models for annular UV disinfection reactors. AIChE Journal, 32(11): 1902-1909,1986.

[9] E. R. Blatchley, III, Numerical modeling of UV intensity: Application to collimated-beam reactors and continuous-flow systems. Water Research, 31(9): 2205-2218, 1997.

[10] K. V. Pareek and A. A. Adesina, Light intensity distribution in a photocatalytic reactor using finite volume. AIChE Journal , 50(6): 1273-1288, 2004.

[11] G. Meinsma and H. Zwart, On $\mathcal{H}_{\infty}$ control for dead-time systems, IEEE Trans. Aut. Control, 45(2): 272-285, 2000.

[12] A. A. Moelja, $\mathrm{H}_{2}$ Control of Systems with I/O Delays. PhD thesis University of Twente, 2005.

[13] S. van Mourik, H. Zwart and K. J. Keesman, Analytic control law for a food storage room. Submitted, 2006.
[14] G. C. Goodwin, S. F. Graebe and M. E. Salgado, Control System Design. Prentice Hall, New Jersey, 2001 